\title{
Correlation of prognostic and predictive indicators in breast cancer patients from the eastern province of Saudi Arabia
}

Haitham A. Kussaibi, $M D, A B$.

\begin{abstract}

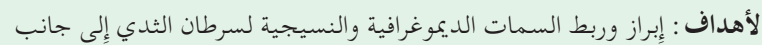

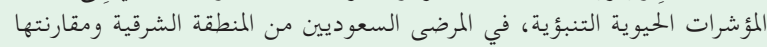
كمجموعة من المرضى غير السعوديين.

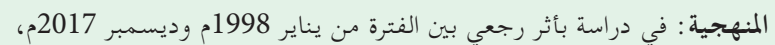

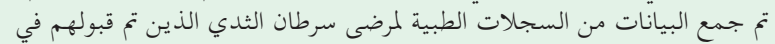

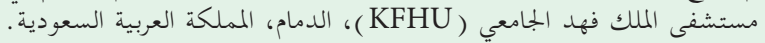
تضمنت المعلومات النتائج الديموغرافية والنسيجية والمكيميائية المناعية .

النتائج : من أصل 482 مريضة بسرطان الثدي ، بلغت نسبة السعوديون $60 \%$ (العبات

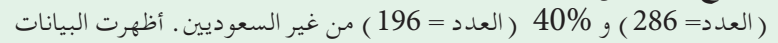

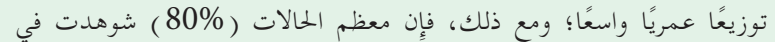

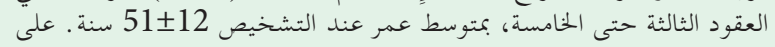

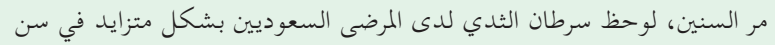

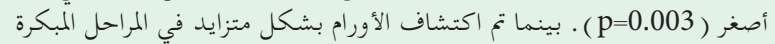

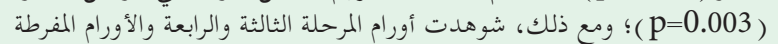

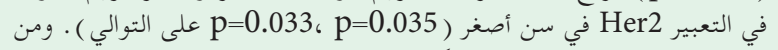
المثير للاهتمام أن هذه النتائج لم تكن مهمة في المرضى غير السعوديين.

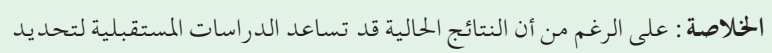

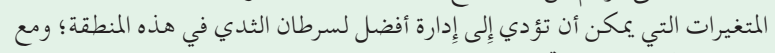

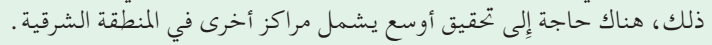

Objectives: To highlight and correlate demographic and histopathological features of breast cancer along with prognostic and predictive biomarkers, in Saudi patients from the Eastern Province and compare it to a cohort of non-Saudi patients.

Methods: In a retrospective study between January 1998 and December 2017, data were collected from the medical records of breast cancer patients who were admitted at King Fahd University Hospital (KFHU), Dammam, Saudi Arabia. The information included demographic, histopathological, and immunohistochemical findings.

Results: Out of 482 breast cancer patients, 60\% (n=286) were Saudis, and $40 \%(n=196)$ were non-Saudis. Data showed a wide age distribution; however, most cases
$(80 \%)$ were seen in the third through fifth decades, with a median age at diagnosis of $51 \pm 12$ years. Over years, breast cancer in Saudi patients was increasingly seen at a younger age $(p=0.003)$. While tumors were increasingly detected at earlier stages $(p=0.003)$; however, stage III $\&$ IV tumors $(p=0.033)$ and human epidermal growth factor receptor- 2 overexpressed tumors $(p=0.035)$ were more frequently seen at a younger age. Interestingly, these associations were not significant in non-Saudi patients.

Conclusion: Although, the current findings might help future studies to identify variables that could lead to better management of breast cancer in this region; however, a wider investigation including other centers in the eastern province is needed.

Keywords: breast cancer, molecular typing, prognostic factors, cancer biomarkers, cancer staging

Saudi Med J 2021; Vol. 42 (3): 293-298 doi: 10.15537/smj.2021.42.3.20200711

From the Pathology Department, College of Medicine, Imam Abdulrahman bin Faisal University, Dammam, Kingdom of Saudi Arabia.

Received 1st December 2020. Accepted 14th January 2021.

Address correspondence and reprint request to: Dr. Haitham A. Kussaibi, Pathology Department, College of Medicine, Imam Abdulrahman bin Faisal University, Dammam, Kingdom of Saudi Arabia.E-mail: hkussaibi@iau.edu.sa

ORCID ID: http://orcid.org/0000-0002-9570-0768

$\mathrm{B}$ reast cancer is the leading cancer in Saudi women, with a steady increase in incidence rate from $19.9 \%$ in 2001 to $30.4 \%$ in 2014 . This rise has been documented in many developed countries worldwide. A recent international study revealed an increase in breast cancer (from 870.000 to 1.937 .000 ) between 1990 and 2017, with the age-standardized incidence rate climbing from 39.2 to 45.9 per 100.000. The shift was mainly found in developing countries; many developed 
countries (for example, USA and UK) showed a decrease in incidence rate. ${ }^{1}$ The mean age at diagnosis in Saudi Arabia is around 50 years, which is different from that reported in developed countries such as the USA, where breast cancer represents $22 \%$ of all newly diagnosed cancers in women with an average age of 60-65 years. ${ }^{2,3}$ Regional variation in breast cancer (for example, biology, prognosis) due to socioeconomic status, race, and ethnicity is well known worldwide. Certain regional disparities in breast cancer are underlined by genetic predispositions such as BRCA1, 2 mutations, which might be reflected in distinct morphological features. ${ }^{4}$ The Eastern Province has, among other KSA regions, the highest overall age-standardized incidence rate (32.6 per 100,000 women). ${ }^{5}$ Between 2009 and 2014, an annual screening program for women $\geq 40$ years old was established in the Eastern Province. ${ }^{6}$ Among 8,061 screened women, breast cancer was detected in $47(0.6 \%)$, of which $70 \%$ had T1 lesions. ${ }^{6}$

Herein, we aim to highlight and correlate demographic and histopathological features of breast cancer along with prognostic and predictive biomarkers, in Saudi patients from the Eastern Province and compare it to a cohort of non-Saudi patients.

Methods. This is a retrospective study based on data of breast cancer patients, retrieved from the medical records over a 20-year period from January 1998 until December 2017. The current study was conducted according to the principles of Helsinki Declaration.

Ethical approval is granted by the Standing Committee on Research Ethics on Living Creatures (SCRELC), Institutional Review Board at Imam Abdulrahman Bin Faisal University (IRB-2020-1-262).

To explore the previous related research, we searched the PubMed database for the recent publications about breast cancer in KSA and worldwide, using the appropriate keywords.

Data of 482 breast cancer patients who were admitted at King Fahd University Hospital (KFHU) during a 20-year period (1998-2017) were collected from medical records. Data included age at diagnosis, nationality, city of residence, tumor (type, grade, stage), lymph node status, and immunohistochemical biomarkers estrogen receptors (ER), progesterone

Disclosure. Authors have no conflict of interests, and the work was not supported or funded by any drug company. receptor (PR), human epidermal growth factor receptor-2 (HER-2), and Ki67.

Inclusion criteria were all-female patients from the Eastern Province admitted at KFHU with invasive mammary carcinoma. Exclusion criteria were patients with missing indispensable data.

Among the 482 breast cancer patients, 60\% $(\mathrm{n}=286)$ were Saudis, while $40 \%(\mathrm{n}=196)$ were non-Saudis. The non-Saudi patients included 35 Filipinos, 30 Egyptians, 21 Yemenis, 18 Syrians, 15 Sudani, 14 Jordanians, 12 Pakistanis, 11 Indonesians, 9 Indians, 6 Palestinians, 5 Lebanese, 3 Bahraini, and 17 patients of other nationalities. The patients, either Saudi or non-Saudi, came from different cities in the Eastern Province, mainly from Khobar and Dammam, followed by Qatif, Hufuf, Dhahran, Hafr Albatin, and Jubail.

Age at diagnosis was classified by decades. Patient nationality was categorized into Saudi or non-Saudi. Tumor types included invasive ductal carcinoma (NOS), invasive lobular carcinoma, or other. Concerning the tumor grading, we followed the modified SBR system (grade I, II, or III); for tumor staging, we followed the TNM/AJCC system: AJCC Stage I, II, or III. ER and PR results were classified into 2 categories: positive or negative. Her 2 results were classified as positive when they were determined as $3+$ by IHC or amplified by fluorescence in situ hybridization (FISH). Estrogen receptors, PR and Her2 scoring followed American Society of Clinical Oncology (ASCO)/College of American Pathologists (CAP) guidelines. Ki67 results were reported as negative, low (1-10\%), intermediate (10-20\%), or high (> 20\%) proliferative index.

The molecular classification was based on immunohistochemistry results, we followed the most updated recommendation; ${ }^{7}$ luminal $\mathrm{A}(\mathrm{ER}+, \mathrm{PR}+$ Her2, and Ki67\% <20\%), luminal B (ER+, PR \pm , Her2, and $\mathrm{Ki} 67 \%>20 \%$ ), luminal Her2 (Her2+ along with $\mathrm{ER}+$ and/or PR+), Her2 enriched (Her2+ while both $\mathrm{ER}$ and $\mathrm{PR}$ are negative), triple-negative (all the 3 biomarkers are negative), and basal-like (when CK5/6 and/or EGFR are positive in triple-negative cancers).

Statistical analysis. Statistical analysis has been performed by IBM SPSS Statistics for Windows, version 26 (IBM Corp., Armonk, N.Y., USA). We utilized for the correlation analysis, the Pearson coefficient. The correlation was considered significant at a p-value $\leq 0.050$.

Results. Data showed a wide age distribution; however, $80 \%$ of patients were $<60$-year-old. The median age at diagnosis was $51 \pm 12$ years in both groups. Over years (1998 to 2017), breast cancer was increasingly seen at a younger age, with a significant 
correlation in Saudi patients $(p=0.003)$; however, that association was not significant in non-Saudis $(p=0.229)$ (Figure 1).

Histopathological features of breast cancer. The most common type was invasive ductal carcinoma (NOS) followed by invasive lobular carcinoma. Most tumors were of grade II followed by grade III. These features were comparable between Saudis and non-Saudis. Approximately 25\% of tumors in Saudi patients, were in stage III and IV, while $62 \%$ in stage II. Non-Saudi patients were seen more frequently in the advanced stage (35\% in stage III and IV and 50\% in stage II) (Table 1). Furthermore, over the 20-year study period, breast cancer was increasingly of lower stages in Saudi patients $(p=0.003)$; rather than non-Saudi patients $(p=0.213)$ (Figure 2). However, tumors in advanced stage were more frequent in younger age in Saudi patients with a significant correlation ( $p=0.033)$; no such association was seen in non-Saudi patients ( $p=0.694)$.

Otherwise, no significant association was found between tumor stage (except with tumor size and lymph node status) and other parameters in the study.

Prognostic and predictive immunohistochemical biomarkers. The negativity rate in Saudi patients for ER was $42.7 \%$, PR 55\%, and Her2 74.7\%. Comparable results were found in non-Saudis. Ki67 was high $(>20 \%)$ in $55 \%$ of Saudi and $60 \%$ of non-Saudi patients (Table 2). A significant negative relationship between Her 2 expression and patient age at diagnosis (Her2 overexpression was more frequently seen in younger age) was noticed in Saudi patients $(p=0.035)$. Interestingly, this association was not significant in non-Saudi patients $(p=0.540)$ (Table 3 ).

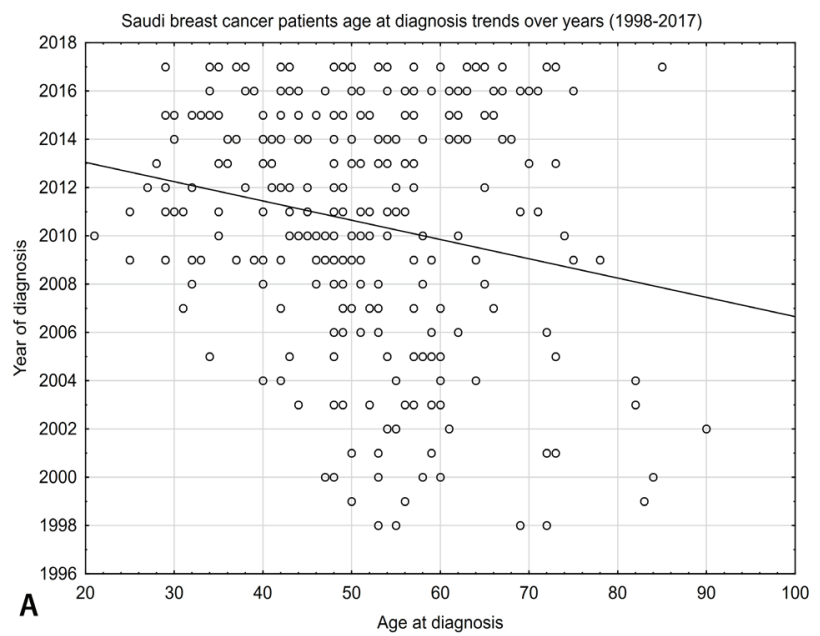

Molecular subtypes. Luminal B was the most frequent among Saudi patients (26.2\%), followed by triple-negative (including basal-like) (25.5\%), luminal A (23.4\%), and Her2-enriched (13.3\%). The least common was the luminal-Her2 type (11.5\%). Comparable findings were observed in non-Saudi patients except for the triple-negative type, which was more frequent $(33.6 \%)$, and the Her2-enriched type which was less frequent (7.9\%) (Table 2).

Over the 20-year period, breast cancer was increasingly of luminal types $(p=0.000)$ in both groups Saudis and non-Saudis (Figure 3).

Table 1 - Breast cancer type, grade, and stage among Saudi and non-Saudi patients.

\begin{tabular}{lrr}
\hline Variables & \multicolumn{2}{c}{ Nationality } \\
& Saudis & Non-Saudis \\
& & \\
\hline Cancer type & & \\
$\quad$ Ductal & $261(91.3)$ & $185(94.4)$ \\
$\quad$ Lobular & $20(7.0)$ & $9(4.6)$ \\
Other & $5(1.7)$ & $2(1.0)$ \\
Cancer grade & & \\
I & $25(8.7)$ & $14(7.1)$ \\
II & $156(54.5)$ & $111(56.6)$ \\
III & $105(36.7)$ & $71(36.2)$ \\
Cancer stage & & \\
I & $26(12.6)$ & $21(14.7)$ \\
II & $129(62.3)$ & $72(50.3)$ \\
III & $51(24.6)$ & $48(33.6)$ \\
IV & $1(0.5)$ & $2(1.4)$ \\
\hline
\end{tabular}

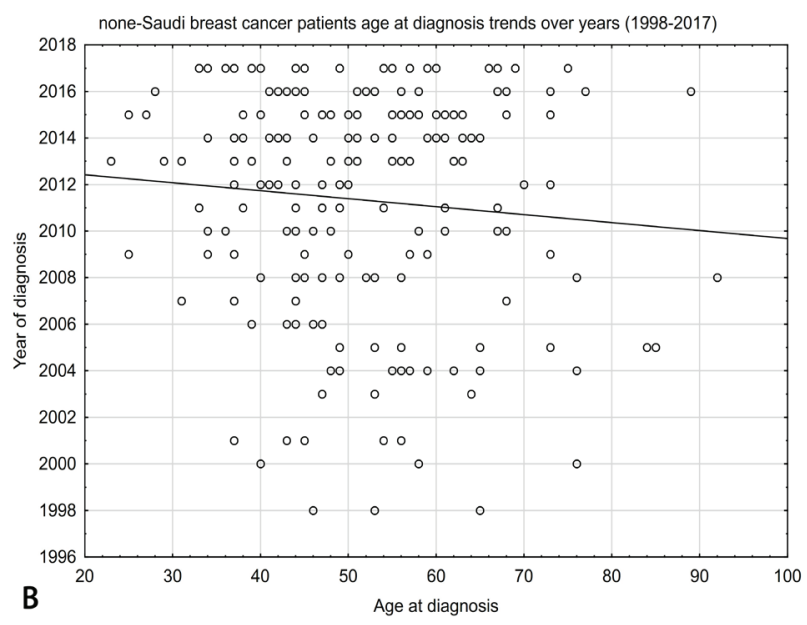

Figure 1 - Breast cancer patients age at diagnosis trends over years (1998-2017) A) Saudi and B) none-Saudi. 

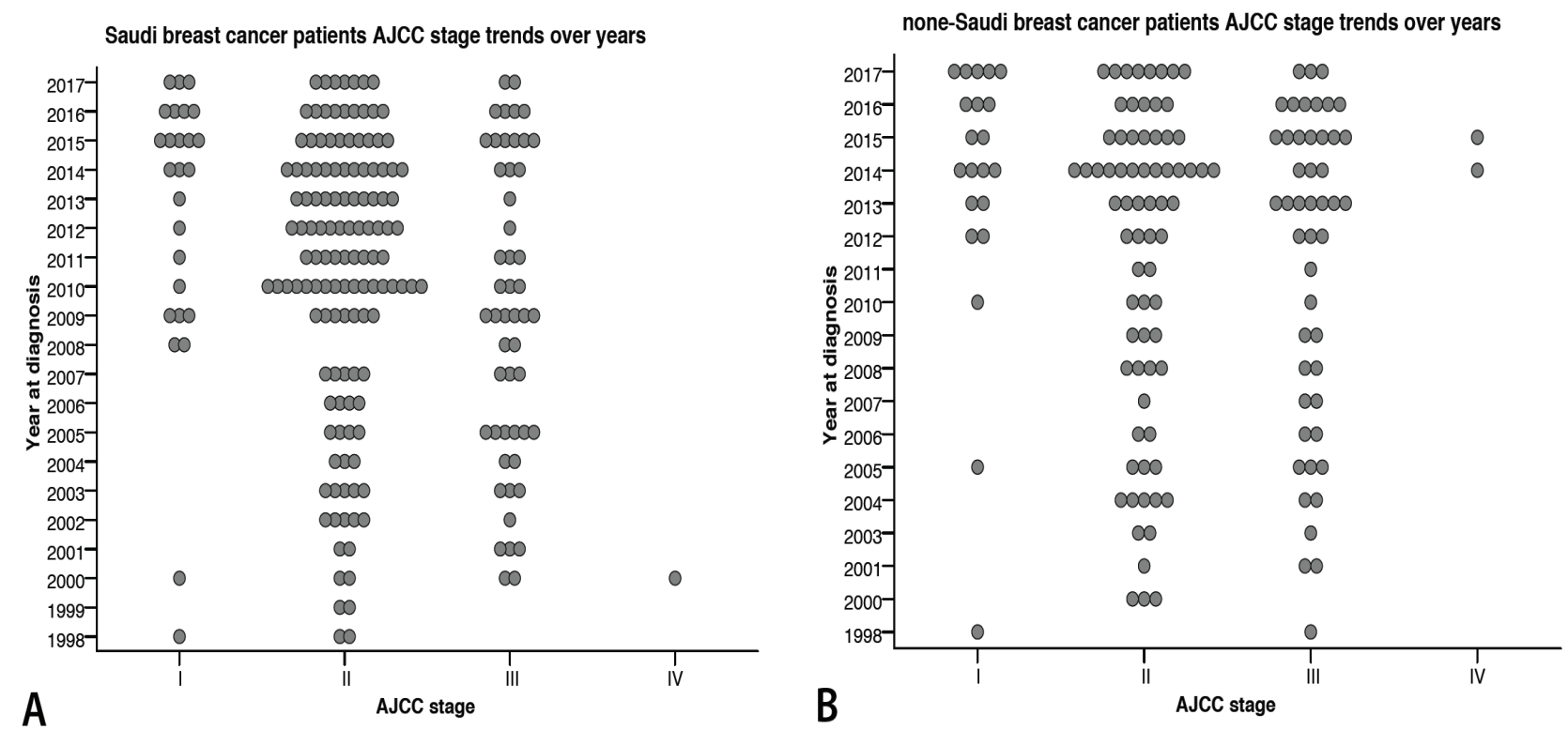

Figure 2 - Breast cancer AJCC stage trends over years (1998-2017) A) Saudi and B) none-Saudi.

Table 2 - Prognostic and predictive biomarkers and molecular subtypes for breast cancer patients.

\begin{tabular}{lrr}
\hline $\begin{array}{l}\text { Biomarkers and molecular } \\
\text { subtypes }\end{array}$ & Saudi & Non-Saudi \\
\hline $\begin{array}{l}\text { ER status } \\
\text {-ve } \\
\text { +ve }\end{array}$ & $120(42.7)$ & $64(45.7)$ \\
PR status & $161(57.3)$ & $76(54.3)$ \\
-ve & & \\
+ve & $154(55.0)$ & $79(56.4)$ \\
Her2 status & $126(45.0)$ & $61(43.6)$ \\
-ve & & \\
+ve & $204(74.7)$ & $106(77.4)$ \\
Ki67 & $69(25.3)$ & $31(22.6)$ \\
Low 1-10\% & & \\
Intermediate 11-20\% & $61(33.7)$ & $27(32.9)$ \\
High >20\% & $21(11.6)$ & $6(7.3)$ \\
Molecular type & $99(54.7)$ & $49(59.8)$ \\
Luminal-A & & \\
Luminal-B & $67(23.4)$ & $33(23.6)$ \\
Luminal-Her2 & $75(26.2)$ & $31(22.1)$ \\
Her2-enriched & $33(11.5)$ & $18(12.9)$ \\
Triple-negative & $38(13.3)$ & $11(7.9)$ \\
Basal-like & $58(20.3)$ & $40(28.6)$ \\
\hline & $15(5.2)$ & $7(5.0)$ \\
\hline
\end{tabular}

-ve: negative, +ve: positive, ER: estrogen receptors, PR: progesterone receptor, Her2: human epidermal growth factor receptor-2
Table 3 - Human epidermal growth factor receptor-2 positive breast cancer: comparison between Saudi and nonSaudi in relation to age at diagnosis.

\begin{tabular}{lcc}
\hline Age at diagnosis & Saudi & Non-Saudi \\
\hline $21-30$ & 7.2 & 3.2 \\
$31-40$ & 24.6 & 9.7 \\
$41-50$ & 21.7 & 35.5 \\
$51-60$ & 33.3 & 35.5 \\
$61-70$ & 10.1 & 6.5 \\
$71-80$ & 1.4 & 9.7 \\
$>80$ & 1.4 & 0.0 \\
\hline
\end{tabular}

Discussion. The current study aims to disclose any significant features of breast cancer in this region as all similar studies were, either outdated or conducted in another region of KSA.

The median age at diagnosis of 51-year is slightly higher than found in many studies in KSA. Furthermore, breast cancer over years was increasingly seen in younger ages in Saudi patients. A study by Saggu et $\mathrm{al}^{8}$ based on 15 years of data (1990-2014) obtained from the Saudi cancer registry, revealed that breast cancer represented 


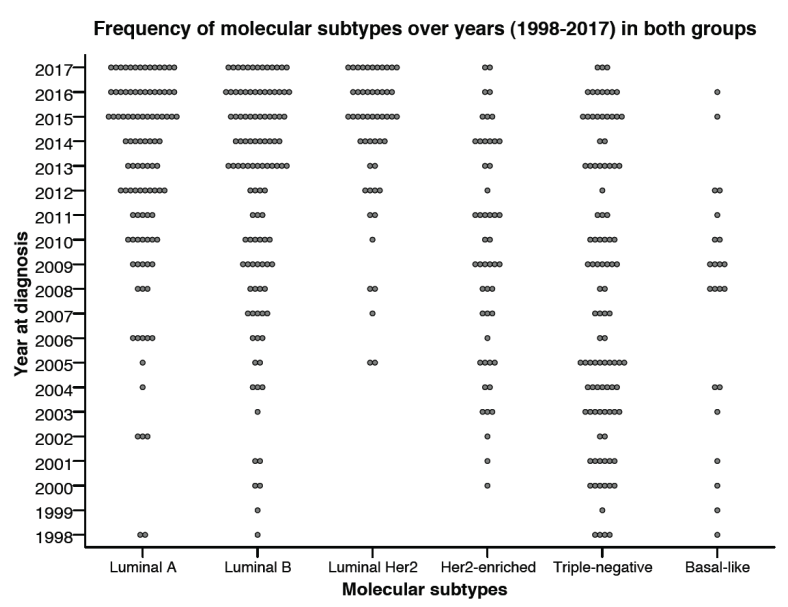

Figure 3 - Frequency of breast cancer molecular subtypes over years (1998-2017) in both groups.

$27 \%$ of all female malignancies and occurred at a younger age (median 48 years) than in developed countries; cases between 1990 and 2010 steadily increased. Another Saudi study carried out on 359 patients from the Riyadh region revealed a mean age at diagnosis of 49.8 years. $^{9}$ A 2018 study on 224 patients, also from the Riyadh region, revealed a younger age distribution of breast cancer than was found in Western nations. However, this was not associated with worse outcomes; overall survival for 10 years was $87 \%$ in all age groups. ${ }^{10}$ Comparable findings were seen in many Middle Eastern countries. In contrast, the mean age at diagnosis of breast cancer is much higher (60-65 years) in developed countries. ${ }^{1}$

Histopathological features of breast cancer. Although the frequency of breast cancer seen in the advanced stage was remarkably higher in non-Saudi patients in comparison with Saudi patients, tumors in the advanced stage were more frequent in younger age in Saudi patients. A significant correlation was noticed between tumor stage and age at diagnosis in Saudi rather than non-Saudi patients. A similar finding reported by Althubiti et al; breast cancer in Saudi Arabia (KSA) is more frequently seen in an advanced stage (III) at a young age ( $46 \pm 11$ years), which is far younger than that reported in western countries (60-65 years). ${ }^{5}$

However, the current study showed that breast cancer in Saudi patients, over years, was increasingly seen in a lower stage. This might be explained by the improvement in the early detection and management of cancer in KSA.

Prognostic and predictive immunohistochemical markers. According to ASCO/CAP guidelines, the percentage of ER-negative breast cancer should not exceed 30\% (20\% for postmenopausal versus 35\% for premenopausal, and up to $10 \%$ in Grade I tumors). ${ }^{11} \mathrm{In}$ our patients, the negativity rate of ER was about $10 \%$ higher than the published benchmark in both groups. While this concurs with the high percentage of Grade III cancers in the current series; however, an annual quality control system with a continuous calibration of the scoring methodology is already in place.

Although the Her2 positivity rate of $25.3 \%$ was within the published benchmarks (10-25\%), ${ }^{12}$ statistical analysis revealed a significant negative relationship between Her 2 expression and patient age at diagnosis (Her2 overexpression was more frequently seen in younger ages), in Saudi patients rather than non-Saudis. Her2-positive breast cancer in patients <40-year-old was much more frequent in Saudi than non-Saudi patients (32\% versus 13\%). A Japanese study on 25,898 patients revealed that young patients $(<35$ years) were associated with an advanced TNM stage and aggressive characteristics, including Her2-positivity, compared with older patients. ${ }^{13}$

In $55-60 \%$ of breast cancer in our series, the Ki67 proliferative index was high $(>20 \%)$, which is well correlated ( $p=0.003$ ) with the high frequency of Grade II and III tumors $(-55 \%$ and $-37 \%)$. However, no significant correlation was seen between Ki67 and patient age at diagnosis $(p=0.194)$. A study from the Al-Madinah region reported Ki67 $>25 \%$ in $73.9 \%$ of the 115 studied patients. ${ }^{14}$ However, the cut-off point value of high Ki67 still controversial. ${ }^{15}$

Molecular subtype frequency. Our results showed that among Saudi patients, luminal-B, triple-negative, and luminal-A showed a close frequency, while luminal Her2 and Her2- enriched were the least frequent. Furthermore, over years, breast cancer was increasingly of luminal subtypes with a lower grade in both groups. However, no significant correlation was found between molecular subtype and age at diagnosis. A Saudi study on 740 breast cancer cases from the western region found that luminal-A was the most common subtype, and the Her2 subtype was the least common. ${ }^{16}$ Furthermore, triple-negative and Her2 subtypes were seen in women younger than 50 years of age and were associated with higher grade and stage. ${ }^{16}$ Similar findings were reported on 359 breast cancer patients from the Riyadh region between 2010 and 2014 (luminal A: 58.5\%, triplenegative: $14.8 \%$, luminal B: $14.5 \%$, and Her2+: $12.3 \%) .{ }^{9}$ These variations in the frequency of different subtypes reflect the controversy in the molecular classification of breast cancer worldwide. 
In conclusion, our data revealed that breast cancer in the Eastern Province of KSA had similar characteristics to those in other regions of KSA and many other developing countries. However, there was a difference in the distribution of molecular subtypes and the Her2 expression frequency among eastern Saudi women, especially in young women. Although, the current findings might help future studies to identify variables that could lead to better management of breast cancer in this region; however, a wider investigation including other centers in the Eastern Province is needed.

Acknowledgment. The author would like to thank Cambridge Proofreading LLC for English language editing.

\section{References}

1. Chen Z, Xu L, Shi W, Zeng F, Zhuo R, Hao X, et al. Trends of female and male breast cancer incidence at the global, regional, and national levels, 1990-2017. Breast Cancer Res Treat 2020; 180: 481-490.

2. Chaudhri E, Fathi W, Hussain F, Hashmi SK. The Increasing Trends in Cases of the Most Common Cancers in Saudi Arabia. J Epidemiol Glob Health 2020; 10: 258-262.

3. Babiker S, Nasir O, Alotaibi SH, Marzogi A, Bogari M, Alghamdi T. Prospective breast cancer risk factors prediction in Saudi women. Saudi J Biol Sci 2020; 27: 1624-1631.

4. Al-Moghrabi NM. BRCA1 promoter methylation in peripheral blood cells and predisposition to breast cancer. J Taibah Univ Med Sci 2017; 12: 189-193.

5. Althubiti MA, Nour Eldein MM. Trends in the incidence and mortality of cancer in Saudi Arabia. Saudi Med J 2018; 39: 1259-1262.

6. Al Mulhim FA, Syed A, Bagatadah WA, Al Muhanna AF. Breast cancer screening programme: experience from Eastern province, Saudi Arabia. East Mediterr Health J 2015; 21: 111-119.
7. Tang P, Tse GM. Immunohistochemical Surrogates for Molecular Classification of Breast Carcinoma: A 2015 Update. Arch Pathol Lab Med 2016; 140: 806-814.

8. Saggu S, Rehman H, Abbas ZK, Ansari AA. Recent incidence and descriptive epidemiological survey of breast cancer in Saudi Arabia. Saudi Med J 2015; 36: 1176-1180.

9. Alnegheimish NA, Alshatwi RA, Alhefdhi RM, Arafah MM, AlRikabi AC, Husain S. Molecular subtypes of breast carcinoma in Saudi Arabia. A retrospective study. Saudi Med J 2016; 37 : 506-512.

10. Alabdulkarim B, Hassanain M, Bokhari A, AlSaif A, Alkarji H. Age distribution and outcomes in patients undergoing breast cancer resection in Saudi Arabia. A single-institute study. Saudi Med J 2018; 39: 464-469.

11. Allison KH, Hammond MEH, Dowsett M, McKernin SE, Carey LA, Fitzgibbons PL, et al. Estrogen and Progesterone Receptor Testing in Breast Cancer: ASCO/CAP Guideline Update. J Clin Oncol 2020; 38: 1346-1366.

12. Wolff AC, Hammond MEH, Allison KH, Harvey BE, Mangu PB, Bartlett JMS, et al. Human epidermal growth factor receptor 2 testing in breast cancer: American Society of Clinical Oncology/College of American Pathologists Clinical Practice Guideline Focused Update. J Clin Oncol 2018; 36: 2105-2122.

13. Kataoka A, Iwamoto T, Tokunaga E, Tomotaki A, Kumamaru $\mathrm{H}$, Miyata $\mathrm{H}$, et al. Young adult breast cancer patients have a poor prognosis independent of prognostic clinicopathological factors: a study from the Japanese Breast Cancer Registry. Breast Cancer Res Treat 2016; 160: 163-172.

14. Elkablawy MA, Albasri AM, Mohammed RA, Hussainy AS, Nouh MM, Alhujaily AS. Ki67 expression in breast cancer. Correlation with prognostic markers and clinicopathological parameters in Saudi patients. Saudi Med J 2016; 37: 137-141.

15. Al Nemer A. The performance of Ki-67 labeling index in different specimen categories of invasive ductal carcinoma of the breast using 2 scoring methods. Appl Immunohistochem Mol Morphol 2017; 25: 86-90.

16. Al-Thoubaity FK. Molecular classification of breast cancer: A retrospective cohort study. Ann Med Surg (Lond) 2020; 49: 44-48. 